\title{
La Universidad en América Latina y sus perspectivas
}

\author{
Henning Jensen Pennington \\ Ph.D., Rector Universidad de Costa Rica. Presidente de la Unión \\ de Universidades de América Latina y el Caribe
}

\begin{abstract}
El concepto de autoridad que corresponde y acompaña a un director o a un maestro en un hogar de estudiantes universitarios no puede apoyarse en la fuerza de disciplinas extrañas a la sustancia misma de los estudios. La autoridad, en un hogar de estudiantes, no se ejercita mandando, sino sugiriendo y amando: enseñando.
\end{abstract}

Las palabras que acabo de citar son conocidas. Son de la juventud argentina de Córdoba, y tienen cien años de haber sido plasmadas en el Manifiesto Liminar cuyas bases de autonomía y progreso inspiraron la creación de las universidades latinoamericanas en las décadas siguientes. Aún hoy mantienen su vigencia por la vastedad de sus alcances y la precisión de sus críticas.

"La idea de libertad es inspiradora. Pero, ¿qué significa? Si eres libre en un sentido político, pero no tienes comida, ¿qué es eso? ¿La libertad de morir de hambre?"

Si en la cita antedicha cambiáramos "libertad" por "autonomía", y "comida" por "recursos", nos daríamos cuenta de que la frase se aplica perfectamente al contexto académico en que vivimos. La frase -pronunciada por la docente, filósofa y activista Angela Davis- tiene su referencia original en la constante denuncia contra la xenofobia, el racismo y el machismo de que sufren las mujeres afroamericanas. Sin demeritar este contexto -y al contrario, pretendiendo ampliar su alcance- es inevitable considerar que no se puede afirmar la defensa de derechos y valores, si por otra parte se reducen o eliminan los medios para realizarlos.

Decidí iniciar este texto con estas palabras, precisamente para evocar esa razón de ser de las instituciones de educación superior. En síntesis, ninguna universidad podría llamarse tal cual si no fuera por su esencia: sus estudiantes. Y no podría llamarse así, tampoco, si su comunidad universitaria no tuviera libertad de pensamiento. 
La libertad se encuentra en el corazón mismo de la universidad como institución cultural. La universidad no puede quedar reducida a ser sólo transmisora de conocimientos, pues requiere que todos se sientan libres de expresarse, de cuestionar, de criticar, de investigar y de proponer, ya que así es como se forjan los procesos de enseñanza-aprendizaje que se dan en la formalidad de las aulas, pero inclusive en el trato cotidiano que se da a cualquier nivel en el marco de una comunidad abierta y solidaria.

No siempre este intercambio en la universidad latinoamericana ha sido de esta manera.

La autonomía universitaria fue una conquista democrática estudiantil alcanzada en 1918 en la conocida Reforma de Córdoba, pero no estuvo aislada de un contexto social que aspiraba y reclamaba libertad e igualdad. Esta aspiración de independencia ha permanecido viva a lo largo de más de cien años a pesar de los regímenes autoritarios que han azotado las tierras latinoamericanas y caribeñas. La voz de los estudiantes cordobeses ha interpelado generación tras generación y sus ideas han inspirado la vocación de libertad de la universidad latinoamericana.

Han sido movimientos que revolucionaron la universidad para convertirla en una institución autónoma al servicio de todos los sectores sociales. Los movimientos estudiantiles inauguraron lo público en el ámbito de la educación superior en América Latina, transformando las universidades en entidades autónomas en su gestión, y en la difusión y generación de conocimiento científico.

En definitiva, la conquista de la autonomía universitaria fue una gesta democrática para las universidades y las sociedades. Y fue una conquista que se dio desde el corazón de una universidad: su movimiento estudiantil.

Esta perspectiva no debe dejar de ser recurrente entre quienes laboramos para una institución de educación superior. En cualquier ámbito, la universidad es de, por y para, sus estudiantes.

Quienes hemos tenido el privilegio de abocarnos a la docencia y la investigación, sabemos bien cuánto potencial se reúne en los jóvenes, ávidos de conocimiento y de salir a ponerlo en práctica. No es de extrañar entonces que la juventud cordobesa tomara una parte protagónica en remover las bases aristocráticas de la universidad e inaugurar la posibilidad del florecimiento de las universidades públicas con libertad de pensamiento y compromiso social.

En efecto, en su pasado prerreformista, la universidad lidió con las doctrinas religiosas que le imponían una actuación dogmática, como también lo hizo contra los poderes privados y autocráticos establecidos en la sociedad y el Estado. Los movimientos estudiantiles crearon a través de la constitución de la universidad pública, las bases democráticas en momentos que las sociedades no erradicaban el espíritu elitista y aristocrático de la época.

Este movimiento que nació siendo argentino, pero rápidamente se propagó como una llama para convertirse en un movimiento latinoamericano, declaraba su empeño por la libertad e igualdad, por el logro de la participación estudiantil en la gestión universitaria, por la extensión cultural de los universitarios, y por la difusión del pensamiento nacional, entre otros aspectos. 
La conquista de la autonomía universitaria convulsionó las sociedades latinoamericanas y creó las bases de una nueva época. Se había conquistado un derecho y se estaba ante un escenario distinto en las relaciones entre la educación superior y la sociedad. Se había gestado la ruptura del poder autocrático con el pensamiento. La autonomía reveló el antagonismo en el sistema social imperante que no se resolvió con su formalización jurídica, pero evidenció y visibilizó la necesidad de limitar tendencias, tensiones o agresiones contra la universidad.

La autonomía universitaria ha sido calificada de especial y completa, pues establece un nivel de independencia que no disfruta ninguna otra institución en la estructura y el funcionamiento del Estado, y supone libertad de jerarquía frente a los supremos poderes. La autonomía le es reconocida a la universidad en razón de sus funciones, objetivos y valores; es decir, por los fines distintivos que la sociedad le ha confiado y cuya ejecución sería incompleta o deficiente sin esa condición autonómica.

Entre esos fines particulares se encuentran la búsqueda de la verdad y la belleza, el cultivo de las artes y las letras, la generación y la transmisión de conocimiento en todos los campos, la crítica reflexiva y el análisis objetivo de la realidad social en sus diferentes dimensiones, y la acción solidaria con su entorno. Estos propósitos no son exclusivos de la universidad, pero le han sido encomendados de manera especial, por lo que le corresponde a ella procurar su realización con decisión y excelencia.

Esta libertad no es una condición que le haya sido otorgada a la institución universitaria de manera gratuita o impensada. En realidad esta independencia ha sido resultado del desarrollo político y representa un logro de la evolución cultural.

Todo esto le imprime a la universidad un sello específico que la compromete con el despliegue de una lógica de funcionamiento diferente a aquellas otras esferas sociales en que rigen mecanismos de regulación social como el poder y el dinero. Acostumbramos a llamar a esa lógica una "lógica académica”, es decir, que es acorde con los elevados fines que la sociedad espera y requiere que la universidad cumpla.

Esta libertad especial no es un atributo consumado por siempre, sino que tiene que ser actualizado y conquistado de manera renovada, en razón de que la libertad constituye un entramado de posibilidades históricas y cambiantes.

La universidad se debate con frecuencia entre la autonomía y la heteronomía, la cual no es impuesta sólo desde fuera. Esto obliga a las comunidades académicas a la práctica reflexiva y autocrítica de sus normas, y las actividades que realiza en procura de sus objetivos: es por eso que la evaluación permanente y la rendición de cuentas constituyen dimensiones sustanciales e irrenunciables de la vida de las instituciones de educación superior pública.

La autonomía universitaria no es un valor absoluto ni solitario. Debe complementarse con la responsabilidad, es decir, la prescripción de que todas las actividades académicas obedezcan a una orientación basada en principios éticos, ante sí misma y de cara a la sociedad. El descuido o abandono de esta dimensión frena la consecución de sus nobles fines y subyuga a la universidad a objetivos meramente instrumentales o funcionales. 
Más de cien años después de la Reforma de Córdoba, nos encontramos en un punto de inflexión importante acerca de la universidad latinoamericana que añoramos: como sociedades en desarrollo y con aspiraciones a convertirnos en economías basadas en el conocimiento. En donde las poblaciones se empoderen de sus decisiones informadas, y los gobiernos sean reflejo de la propia definición de desarrollo que se geste de manera participativa.

Las universidades tienen un papel fundamental en este camino, pero es necesario emprender procesos de autoevaluación, planificación a largo plazo, diálogo interno y negociación, para preservar su carácter imprescindible para la sociedad.

En un contexto muy complejo y cambiante, en medio de grandes logros y desafíos, la coyuntura es particularmente fértil para el análisis de lo que se ha hecho y de lo que se quiere hacer, de nuestras realidades y nuestros sueños. En este contexto quiero enunciar algunas reflexiones sucintas sobre nuestra institución y las tareas que tenemos, y las que se nos avecinan.

La universidad es central y emblemática en la institucionalidad latinoamericana. Ha sido -y es- un pilar en la construcción del estado social de derecho del que nos enorgullecemos, o al que aspiramos. En muchas de nuestras sociedades se trata de las instituciones mejor valoradas por la población, debido a su aporte continuo y significativo al desarrollo nacional y a la calidad de vida de cada país. Este papel y esta valoración conllevan una enorme responsabilidad, tanto de las autoridades superiores de la institución como de la comunidad universitaria en general. Es esa una responsabilidad que debemos fortalecer y asegurar.

La universidad latinoamericana y caribeña se desarrolla en un contexto nacional e internacional no siempre favorable a sus valores y propósitos. Ningún país está exento de vivir situaciones que azucen su institucionalidad, o incluso que comprometan la autonomía universitaria. Pero desde el quehacer de las universidades hay una obligación por ser partícipes de la puesta en común de ideas y voluntades para contribuir a esa mejora del ambiente nacional, para brindar prosperidad y alcanzar el bien común.

La globalización y la imposición de una lógica mercantil asimétrica en las relaciones entre países, y al interior de ellos, han generado un escenario global y nacional donde las inequidades se han incrementado, y los valores de solidaridad, propósito colectivo y de sana relación con el ambiente, se han debilitado sistemáticamente pese a que importantes sectores de la población luchan denodadamente por preservar formas de vida solidarias y humanistas. Lamentablemente, las corrientes ideológicas dominantes en muchos de nuestros países no caminan hacia la misma dirección que los valores esenciales de la universidad latinoamericana.

Estas instituciones deben centrar su aporte académico y social alrededor de la construcción de una sociedad justa y equitativa, donde la excelencia académica, el desarrollo pleno de todas las personas y el buen manejo del patrimonio deben ser las luces que guíen nuestro accionar. Este desiderátum choca con fuertes tendencias que predominan en el escenario político e ideológico nacional e internacional, pues hay quienes ven en estos valores una amenaza a sus proyectos políticos, dedicados a buscar el bienestar de una minoría y a dilapidar de manera irracional el patrimonio 
natural. Hay quienes pretenden debilitar a toda costa a la educación superior pública mediante campañas bien orquestadas y mal intencionadas, y una sistemática desvalorización de las instituciones públicas en la vida de nuestras naciones.

Todas las comunidades universitarias deben tener claro este panorama adverso y enfrentarlo con decisión para seguir construyendo instituciones que contribuyan a un desarrollo regional social y ecológicamente responsable, que procure el desarrollo integral de las personas y no su conversión en mero homo economicus al servicio de la lógica que antepone lo mercantil por sobre lo humano. Lograr este cometido involucra una gran cantidad de tareas de muy diverso tipo.

De cara a los procesos que se imponen en la sociedad global, donde se da una paulatina, pero segura erosión de un concepto de sociedad basado en la solidaridad, en las universidades cultivamos el fin de educar y formar a las nuevas generaciones según los valores de una ciudadanía reflexiva, crítica y propositiva. Lo hacemos en un complejo escenario de objetivos culturales, académicos y sociales, los cuales se materializan en la docencia, la investigación y la acción social, como elementos fundamentales de estas instituciones.

La integración de las tres actividades sustantivas que realizan las unidades académicas y quienes allí trabajan, no es nada sencillo. Por el contrario, se requieren esfuerzos sistemáticos y programas coordinados para que nuestros jóvenes reciban conocimientos actualizados, aprendan procesos avanzados de investigación y, a la vez, junto con sus profesores, pongan ese conocimiento al servicio de la sociedad. Ninguna universidad que se dedique sólo a la docencia puede tener idea de la complejidad de este propósito, la cual se vuelve aún más grande si tomamos en cuenta la diversidad de los sectores sociales involucrados y la rapidez con que evoluciona el conocimiento en todas las áreas y disciplinas.

Es por ello que toda universidad debe poner especial énfasis en la calidad de sus docentes y estudiantes. A este fin sirve la planificación del relevo generacional y la depuración de los procesos y mecanismos de contratación de personal académico, a lo cual debe agregarse de manera complementaria la formación continua del personal académico y administrativo. Como parte de este proceso se requiere fortalecer los programas de posgrado y los estudios interdisciplinarios avanzados, y dirigir las políticas académicas hacia un sistema de méritos con salarios competitivos en el marco nacional e internacional. Las universidades como instituciones de alta cultura, ciencia y tecnología, desarrollan su quehacer en el contexto de una amplia internacionalidad, en la cual predomina la transmisión cruzada de conocimientos de toda naturaleza y de experiencias compartidas. El conocimiento se encuentra ahora más distribuido en el mundo. Las universidades avanzadas son aquellas abiertas a la movilidad multidireccional de docentes y estudiantes, y que realizan proyectos compartidos de alto nivel, abordan conjuntamente temas de gran complejidad, y se nutren de la diversidad cultural en relaciones de respeto y beneficio mutuo.

Ante los cambios acelerados del conocimiento, son deseables las acciones que contribuyan a una mayor flexibilidad y versatilidad curricular, con generación de nuevos programas, integración de áreas diversas y movili- 
dad de estudiantes entre diferentes disciplinas. En el caso de aquellas universidades con representación regional, se deben promover modelos horizontales de regionalización, que articulen la pertinencia territorial con relevancia nacional, acorde con las necesidades del país en general y de las regiones en particular, así como de la ciencia, la tecnología, el arte y las humanidades.

En el contexto de una internacionalidad que hemos de llamar solidaria, en contraste con una globalización que excluye y desintegra, una universidad completa debe consolidar su posición en ese escenario académico, ahora más extenso que nunca antes, dando énfasis a alianzas académicas mediante formación de redes amplias y flexibles que permitan a los grupos universitarios ubicarse en el escenario de la ciencia y la tecnología, así como del arte y las humanidades a escalas regionales y mundiales.

Hasta hace poco tiempo las mejores universidades del mundo estaban ubicadas en Europa y Norteamérica. Ahora hay excelentes universidades en prácticamente todos los continentes. En nuestra América Latina no podemos ser la excepción. Si nuestros países pretenden jugar un papel digno y próspero en el concierto de las naciones del mundo, ya sea en el campo económico, cultural, científico o político; deben disponer de universidades dinámicas y creativas, que cumplan los más altos estándares de calidad y estén insertas en el contexto internacional.

La América Latina de hoy es la más desigual que hemos conocido. La universidad pública en esta región ha sido la vía regia de la movilidad social, un medio de fortalecimiento de la equidad, del combate contra la pobreza y a favor de la generación de oportunidades.

La universidad pública contribuye al desarrollo de capacidades en los distintos sectores de cada país y pone un particular énfasis en sectores sociales vulnerables. La transferencia del conocimiento, como se le ha llamado a lo largo de los años, debe contribuir al desarrollo de una producción social de beneficio colectivo que fomente el bienestar de grupos y comunidades, particularmente las más vulnerables. La universidad pública debe estimular y aprovechar las sinergias e interacciones con otras instituciones nacionales y así cimentar políticas eficaces en esa transferencia del conocimiento a la sociedad. El saber generado en nuestras casas de estudios superiores debe permear toda la sociedad, en toda la nación, pero esa permeabilidad debe ser recíproca: la universidad pública debe aprender de los saberes colectivos de todos los agentes sociales.

Una excelente universidad se fortalece con excelentes estudiantes de todos los estratos sociales. Así como la Organización de las Naciones Unidas se enfoca en "no dejar a nadie atrás" para promover los Objetivos de Desarrollo Sostenible, cada una de nuestras universidades debe pensar en las vías de promover la inclusión y fomentar la equidad, para que sean motor de la sociedad que queremos para nuestros países, a pesar de que las desigualdades de la sociedad -muchas veces- se reflejen en ellas mismas. Y no sólo eso: así como muchas de las instituciones públicas están llamadas a ser partícipes de los planes de desarrollo de los países, es un buen momento también para insertarnos en la misión de nuestras naciones por promover estos objetivos globales, de modo que cada aporte sea visibilizado y sea revestido de la importancia que merece para fomentar el alcance 
de las metas de desarrollo mundiales de la mano de la equidad entre todos los habitantes de esta aldea global.

Esta equidad se repite, también, en el nivel territorial, cuando la universidad tiene una proyección hacia cada rincón de su país. Al profundizar una gestión institucional horizontal y cada vez más descentralizada hacia los territorios, en todos los niveles operativos se fortalecerá un concepto institucional sistémico, unitario, integral y eficiente. Esta articulación orgánica de las líneas de desarrollo académico con las políticas y las acciones estratégicas institucionales contribuirá asertivamente, sin duda, a la inclusión social de sectores de las zonas de influencia ubicados bajo la línea de pobreza.

Pero el alcance de una universidad, tanto a nivel regional como internacional, debe empezar por su propio campus, en cada una de sus sedes. Es decir, cada miembro de la comunidad universitaria debe sentir cómo estas instituciones permean en sus criterios, les otorgan nuevos puntos de vista, y abren oportunidades y posibilidades de desarrollo. Desde algo tan obvio (y, sin embargo, aún muchas veces condicionado) como la garantía de derechos fundamentales en cada institución, hasta la ampliación de servicios que tradicionalmente no se han atendido. Se crea una mayor cohesión universitaria, esencial para que se genere más reflexión en torno al tipo de servicios que se buscan en la institucionalidad pública, y que el Estado debe garantizar.

En un sencillo ejemplo, la humanización del espacio físico en el campus y los aportes a la construcción de un espacio urbano que interactúa con la ciudad circundante, como sucede en muchas ciudades universitarias latinoamericanas, provocan resonancia en la ciudadanía y son parte del discurso político nacional. Estoy seguro de que la preservación cultural y la memoria histórica son equivalentes de este aporte fundamental en la sociedad, en donde el papel relevante de las investigaciones y la defensa de la tradición se conjugan para facilitar que las personas lo defiendan en su diario vivir, y en los servicios que demandan para sí y para sus familias.

En una dimensión diferente los retos económicos en nuestros países son vastos. No sólo por la coyuntura internacional de delicada trascendencia, sino también porque los recursos para la cooperación internacional escasean, y los presupuestos nacionales son cada vez más restrictivos y restringidos. La búsqueda de la sostenibilidad financiera de la institucionalidad no debe escapar a la universidad latinoamericana, puesto que es una de las mejores maneras para demostrar su eficiencia pero también la necesidad de invertir en educación superior, en todas sus dimensiones y alcances, reconociendo la productividad académica y la creatividad artística. Las acciones concretas deben ser transparentes, tomadas con severa y rigurosa decisión, considerando no sólo el futuro inmediato, sino también la subsistencia de la universidad en el tiempo. Esto, además, contribuye a desmitificar la autonomía como un abuso, cuando en realidad implica la capacidad de tomar, precisamente, este tipo de decisiones.

En la declaración de la VII Asamblea General Extraordinaria de la Unión de Universidades de América Latina y el Caribe (UDUAL), rumbo a Conferencia Regional de Educación Superior de 2018; declaramos que la autonomía en esta nueva hora latinoamericana se inscribe en una preserva- 
ción del derecho a la educación, a la igualdad, a los derechos humanos, a la libertad de pensamiento y a la descolonización de los valores y prácticas de apropiación del conocimiento. Por ello defendemos una autonomía que involucra a las universidades en su capacidad de gestión, gobierno y libertades, pero también en su compromiso y su naturaleza de instrumentos de equidad y justicia social.

Retomando los tres pilares sobre los cuales las universidades latinoamericanas han basado su gestión en el último centenario -la docencia, la investigación y la acción social- me permito hacer un breve hincapié en el último. Quisiera retomar un breve extracto del manifiesto de la Universidad de Costa Rica a la luz del centenario de la Reforma de Córdoba, pues considero que se aplica a toda universidad latinoamericana, no sólo por sus particularidades, sino por la propia región diversa y de enorme riqueza en la que se erigen. Cito:

Debemos posicionar en las agendas universitarias acciones concretas para la construcción de una universidad de los saberes, entendida como un espacio de construcción de nuevos conocimientos y caminos de emancipación, que permita profundizar la democracia, desmercantilizar la vida, descolonizar y eliminar el racismo y el etnocentrismo, eliminar todas las formas de sexismo y discriminación, así como la construcción de un concepto de solidaridad basado en el concepto de bien común y en la generación de una calidez y respeto entre las personas de estas hacia la naturaleza.

Estos son algunos de los retos que nos plantea el futuro. Todos ellos deben verse en el contexto de una realidad nacional y regional compleja y contradictoria. El liderazgo institucional que las universidades latinoamericanas han forjado a lo largo de su historia nos conduce a promover una fuerte interacción con sectores sociales y políticos externos, en procesos permanentes de diálogo y negociación, para garantizar presupuestos adecuados y justos para la institución, y fortalecer vínculos de diverso tipo con instituciones, sectores y regiones.

Al enfrentar nuevos retos no debemos caer en la autocomplacencia y la falta de autocrítica. Por el contrario, debemos tener oídos receptivos ante la crítica constructiva, y extender nuestros brazos para recibir los aportes ajenos. La actitud dialógica nos beneficiará como instituciones que son forjadoras de ciudadanía y constructoras de nación. Las universidades no deben perder su misión ni su carácter esencial. 